\title{
Monocyte-Derived Macrophages Modulate Inflammation and Promote Long-Term Functional Recovery in a Mouse Model of Ischemia
}

\author{
Adam Kovacs-Litman ${ }^{1 *}$ and $\odot$ Ilan Vonderwalde ${ }^{2 *}$ \\ ${ }^{1}$ Schulich School of Medicine and Dentistry, Western University, London, Ontario N6A 3K7, Canada, and ${ }^{2}$ Institute of Biomaterials and Biomedical \\ Engineering, University of Toronto, Toronto, Ontario M5S 3E1, Canada \\ Review of Wattananit et al.
}

The dogma of stroke recovery has dramatically changed over the last several decades. It was long believed that neurons were the sole mediator of functional decline and recovery following stroke (Garcia, 1975). More recently, however, the importance of resident astrocytes, oligodendrocytes, and microglia in the pathogenesis and recovery of this disease has become apparent (Mabuchi et al., 2000; Takano et al., 2009; Jin et al., 2010). Wattananit et al. (2016) furthered this understanding by examining the role of immune cells that infiltrate the infarcted region from outside the CNS. Specifically, they showed that monocyte-derived macrophages (MDMs) preferentially migrate to the ischemic site, become involved in the inflammatory response, and facilitate longterm functional recovery. MDMs and other inflammatory mediators may therefore become a therapeutic target in promoting rehabilitation following stroke.

In response to cellular and tissue damage, circulating monocytes migrate to sites of injury and differentiate into macrophages, which participate in the inflammatory response by clearing debris and

Received June 12, 2016; revised Aug. 3, 2016; accepted Aug. 8, 2016. The authors declare no competing financial interests.

${ }^{*}$ A.K.-L. and I.V. contributed equally to this study as co-first authors. Correspondence should be addressed to Mr. Ilan Vonderwalde, $160 \mathrm{Col}$ lege Street, Room 1040, Toronto, Ontario M5S 3E1, Canada. E-mail: ilan.vonderwalde@mail.utoronto.ca.

DOI:10.1523/JNEUROSCI.1906-16.2016

Copyright $\odot 2016$ the authors $\quad 0270-6474 / 16 / 369757-03 \$ 15.00 / 0$ secreting immunomodulators. Depending on the microenvironment at the lesion site, different signaling pathways can give rise to two separate populations of macrophages with distinct phenotypes and function (Italiani and Boraschi, 2014). These two populations have opposing roles in the inflammatory response. Proinflammatory M1 macrophages release chemokines and cytokines to recruit other immune effector cells, whereas anti-inflammatory M2 macrophages, also known as healing or deactivated macrophages, aid in tissue repair homeostasis (David and Kroner, 2011; Prinz and Priller, 2014).

Resident microglia in the CNS, which are also derived from myeloid cells, can similarly be polarized in response to damage. Thus, it is important to be able to identify different cells in the brain to fully understand their contributions to postischemic changes. To address this, the expression of certain markers can be used to identify cell populations. Macrophages and microglia can be distinguished from other cells by their expression of CD11b, and from each other by varied expression of CD45 (Greter et al., 2015). These markers allow scientists to use a variety of techniques, such as flow cytometry and immunohistochemistry, to identify specific cell types.

Using a medial cerebral artery occlusion model of stroke in mice, Wattananit et al. (2016) explored the properties of circulating monocytes and their inflammatory role in stroke recovery. They found a correlation between the number of monocytes in the peripheral circulation and MDMs in the ischemic lesion. Furthermore, many more endogenous MDMs were present in the stroke-affected cerebral hemispheres than in the contralateral hemispheres, suggesting that circulating monocytes are recruited specifically to the ischemic site.

Using flow cytometry, the authors determined that the number of monocytes infiltrating the ischemic region peaks at $3 \mathrm{~d}$, remains significantly higher than baseline at $7 \mathrm{~d}$, and returns to control levels by $14 \mathrm{~d}$ after stroke. This suggests an initial surge in immune cell recruitment following an ischemic event, which decreases over time.

Two subsets of MDMs were identified in the ischemic brain using flow cytometry: a proinflammatory (Ly6C $\mathrm{Cigh}^{\text {heX }} / \mathrm{CR} 1^{\text {low }}$ ) population and an anti-inflammatory (Ly6C $^{\text {low }} / \mathrm{CX} 3 \mathrm{CR} 1^{\text {int }}$ ) population. The proportion of total MDMs that fell into these categories shifted over time. Specifically, a shift from a proinflammatory to an antiinflammatory bias appeared to occur between days 3 and 14 after stroke. The occurrence of this shift was further supported by an increase in anti-inflammatory markers CD206 and Dectin1 within the Ly6C ${ }^{\text {low }} / \mathrm{CX} 3 \mathrm{CR} 1^{\text {int }}$ population between day 3 and day 7 ; the authors did not examine changes in proinflammatory markers at the same times. Because MDMs are a heterogeneous population with different roles in the 
ischemic environment, their phenotype might be determined by environmental cues. It may also be possible that individual cells change phenotype due to external signals (Italiani and Boraschi, 2014). Nevertheless, there seems to be a shift in the inflammatory profile of MDMs following stroke.

By analyzing the expression of CD45 and CB11b, Wattananit et al. (2016) found that, whereas MDMs made up the majority of cells in the ischemic region $3 \mathrm{~d}$ after stroke, microglia accounted for the majority of cells at $7 \mathrm{~d}$ after stroke. The authors also found that microglia have a sustained proinflammatory phenotype at 3 and $7 \mathrm{~d}$ after stroke. In addition, compared with the contralateral hemisphere, stroke-affected hemispheres exhibited significantly higher expression of Ym1, an anti-inflammatory marker, at $3 \mathrm{~d}$ after stroke, higher expression of both proinflammatory (IL-6, TNF $\alpha$, IL$1 \beta$, NOS2) and anti-inflammatory markers (TGF $\beta 1$, Ym1, Cxcl12, CCL22, CD163) $7 \mathrm{~d}$ after stroke, and higher expression of antiinflammatory markers (TGF $\beta 1$, VCAM1) $14 \mathrm{~d}$ after stroke. It is of note that the inflammatory mediators examined are not released exclusively by MDMs, suggesting that other cell types also play a role in the inflammatory response. This is supported by the high expression of Ym1 $3 \mathrm{~d}$ after stroke, a time when MDMs expressed a predominantly proinflammatory phenotype.

To understand the importance of MDMs in the inflammatory environment and functional recovery, Wattananit et al. (2016) depleted monocytes for $10 \mathrm{~d}$ following stroke (when they would normally be at peak levels of infiltration) by administering daily injections of MC-21, a CCR2 antibody, on the day of insult and for the next $3 \mathrm{~d}$. CCR2 mediates monocyte egress from bone marrow to the circulation, and cells expressing it have been shown to preferentially migrate to injured tissue, including the CNS (Yan et al., 2007; Greter et al., 2015). The depletion of monocytes led to a dramatic decrease in the anti-inflammatory marker Ym1 a week after stroke. Interestingly, after circulating monocytes were restored $14 \mathrm{~d}$ after stroke, there was still a decrease in the percentage of monocytes and microglia within the ischemic region. Furthermore, quantitative PCR revealed a decrease in three anti-inflammatory markers (TGF $\beta 1$, TGF $\beta 2$, and CD163), and in NOS2, a proinflammatory marker at $14 \mathrm{~d}$ after stroke.

To investigate how depletion of monocytes for the first week following stroke affected long-term spontaneous recovery, functional assessment was conducted using behavioral tests that measure lateralized sensorimotor integration or forelimb reaching and grasping. Animals in which monocytes had been depleted had sustained deficits that persisted after control animals had recovered. These results suggest that early recruitment of circulating monocytes may play an important role in stroke recovery.

This research expands the scope of our understanding of stroke recovery and its cellular underpinnings. It is clear that MDMs play a role in mediating the inflammatory response in the injured brain following stroke. Future experiments should more fully explore the relative importance of specific proinflammatory and anti-inflammatory factors in stroke recovery. Wattananit et al. (2016) conclude that MDMs transition from a proinflammatory to an anti-inflammatory bias during the first 2 weeks following ischemia. Although this conclusion is supported by their data, it does not paint a full picture of the inflammatory environment within the ischemic lesion as other inflammatory cells, reactive astrocytes, stem cells, and excitotoxic events may also be involved (Liesz et al., 2009; Erlandsson et al., 2011; Faiz et al., 2015). The authors also posit that anti-inflammatory properties of MDMs promote functional recovery. This is consistent with much of the literature and the authors' observation of decreases in anti-inflammatory markers at day 7 and 14 when monocytes were depleted with MC-21. As other studies have considered, it is likely that both proinflammatory and anti-inflammatory factors are necessary for optimal functional recovery (London et al., 2013). The data presented by Wattananit et al. (2016) support the conclusion that, although excessive inflammation may inhibit stroke recovery, some proinflammatory factors may be necessary to attract specific cell populations that facilitate recovery.

In addition, it is important to note that the inflammatory markers reported in the ischemic environment may not be representative of MDM activity. Microglia (and other cell types) can modulate inflammation and may be responsible for some of the markers reported, particularly around $7 \mathrm{~d}$ when microglia are most predominant (Block and Hong, 2005; Erlandsson et al., 2011). Future experiments should seek to isolate inflammatory modulation caused by MDMs and other cells by selectively inhibiting microglia activity.

Some controversy exists surrounding the role of Ly6 $\mathrm{C}^{\text {high }}$ monocytes in stroke, specifically whether they promote or inhibit recovery. A study by Hammond et al. (2014) found that Ly6 $\mathrm{C}^{\text {high }}$ monocytes actually ex- acerbated acute disability following stroke, whereas a study by Chu et al. (2015) found they were protective, corroborating the results of this study. Notably, the study by Hammond et al. (2014) used an intracerebral hemorrhage model of stroke instead of the ischemic stroke model used by Chu et al. (2015) and Wattananit et al. (2016). Despite this admittedly large difference, future studies should seek to reconcile Ly6 $\mathrm{C}^{\text {high }}$ monocytes as a protective or damaging factor in ischemic and hemorrhagic stroke, respectively.

Last, it is critical to note that sex, age, and lesion severity, which were not directly addressed in this study, have previously been shown to influence inflammatory response and recovery following stroke using animal models (Bodhankar et al., 2015; Suenaga et al., 2015). These are important factors to consider for clinical translation of this research.

In conclusion, Wattananit et al. (2016) have demonstrated the role of MDMs in promoting spontaneous functional recovery and modulating the inflammatory response in the strokeinjured brain. Inflammation and the involvement of peripheral immune cells appear to play a larger role in stroke pathogenesis than was previously believed. Further studies are necessary to identify the possibility of targeting inflammatory factors as a novel therapeutic approach for promoting functional recovery after stroke.

\section{References}

Block ML, Hong JS (2005) Microglia and inflammation-mediated neurodegeneration: multiple triggers with a common mechanism. Prog Neurobiol 76:77-98. CrossRef Medline

Bodhankar S, Lapato A, Chen Y, Vandenbark AA, Saugstad JA, Offner H (2015) Role for microglia in sex differences after ischemic stroke: importance of M2. Metab Brain Dis 30:15151529. CrossRef Medline

Chu HX, Broughton BR, Kim HA, Lee S, Drummond GR, Sobey CG (2015) Evidence that Ly6Chi monocytes are protective in acute ischemic stroke by promoting M2 macrophage polarization. Stroke 46:1929-1937. CrossRef Medline

David S, Kroner A (2011) Repertoire of microglial and macrophage responses after spinal cord injury. Nat Rev Neurosci 12:388-399. CrossRef Medline

Erlandsson A, Lin CH, Yu F, Morshead CM (2011) Immunosuppression promotes endogenous neural stem and progenitor cell migration and tissue regeneration after ischemic injury. Exp Neurol 230:48-57. CrossRef Medline

Faiz M, Sachewsky N, Gascón S, Bang KW, Morshead CM, Nagy A (2015) Adult neural stem cells from the subventricular zone give rise to 
reactive astrocytes in the cortex after stroke. Cell Stem Cell 17:624-634. CrossRef Medline

Garcia JH (1975) The neuropathology of stroke. Hum Pathol 6:583-598. CrossRef Medline

Greter M, Lelios I, Croxford AL (2015) Microglia versus myeloid cell nomenclature during brain inflammation. Front Immunol 6:249. CrossRef Medline

Hammond MD, Taylor RA, Mullen MT, Ai Y, Aguila HL, Mack M, Kasner SE, McCullough LD, Sansing LH (2014) CCR2 ${ }^{+}$Ly6Chi inflammatory monocyte recruitment exacerbates acute disability following intracerebral hemorrhage. J Neurosci 34:3901-3909. CrossRef Medline

Italiani P, Boraschi D (2014) From monocytes to M1/M2 macrophages: phenotypical vs. functional differentiation. Front Immunol 5:514. CrossRef Medline

Jin R, Yang G, Li G (2010) Inflammatory mechanisms in ischemic stroke: role of inflammatory cells. J Leukoc Biol 87:779-789. CrossRef Medline
Liesz A, Suri-Payer E, Veltkamp C, Doerr H, Sommer C, Rivest S, Giese T, Veltkamp R (2009) Regulatory T cells are key cerebroprotective immunomodulators in acute experimental stroke. Nat Med 15:192-199. CrossRef Medline

London A, Cohen M, Schwartz M (2013) Microglia and monocyte-derived macrophages: functionally distinct populations that act in concert in CNS plasticity and repair. Front Cell Neurosci 7:3389. CrossRef Medline

Mabuchi T, Kitagawa K, Ohtsuki T, Kuwabara K, Yagita Y, Yanagihara T, Hori M, Matsumoto M (2000) Contribution of micro$\mathrm{glia} / \mathrm{macrophages} \mathrm{to} \mathrm{expansion} \mathrm{of} \mathrm{infarction}$ and response of oligodendrocytes after focal cerebral ischemia in rats. Stroke 31:17351743. CrossRef Medline

Prinz M, Priller J (2014) Microglia and brain macrophages in the molecular age: from origin to neuropsychiatric disease. Nat Rev Neurosci 15:300-312. CrossRef Medline

Suenaga J, Hu X, Pu H, Shi Y, Hassan SH, Xu M,
Leak RK, Stetler RA, Gao Y, Chen J (2015) White matter injury and microglia/macrophage polarization are strongly linked with age-related long-term deficits in neurological function after stroke. Exp Neurol 272:109119. CrossRef Medline

Takano T, Oberheim N, Cotrina ML, Nedergaard M (2009) Astrocytes and ischemic injury. Stroke 40:S8-S12. CrossRef Medline

Wattananit S, Tornero D, Graubardt N, Memanishvili T, Monni E, Tatarishvili J, Miskinyte G, Ge R, Ahlenius H, Lindvall O, Schwartz M, Kokaia Z (2016) Monocyte-derived macrophages contribute to spontaneous long-term functional recovery after stroke in mice. J Neurosci 36:4182-4195. CrossRef Medline

Yan YP, Sailor KA, Lang BT, Park SW, Vemuganti R, Dempsey RJ (2007) Monocyte chemoattractant protein-1 plays a critical role in neuroblast migration after focal cerebral ischemia. J Cereb Blood Flow Metab 27:12131224. CrossRef Medline 